Rheum Dis Clin North Am. 2018 August ; 44(3): xiii-xiv. doi:10.1016/j.rdc.2018.05.001.

\title{
Rheumatic Diseases in Older Adults
}

\author{
James D. Katz, MD [Editors] and \\ National Institutes of Health, National Institutes of Arthritis and Musculoskeletal and Skin \\ Disease, Building 10, Room 10N-311, 9000 Rockville Pike, Bethesda, MD 20892, USA \\ Brian Walitt, MD, MPH [Editors] \\ National Institute of Dental and Craniofacial Research, National Institutes of Health, Building 10, \\ Room 2-1341, 10 Center Drive, Bethesda, MD 20892, USA
}

\begin{abstract}
We are pleased to present this special issue of Rheumatic Disease Clinics of North America that is devoted to Older Adults. It is an extension of our earlier work already published in the cousin series, Clinics in Geriatric Medicine. ${ }^{1}$ To this end, we have reassembled and enhanced the broad range of expertise that will now serve to highlight the latest diagnostic and therapeutic information in the field. Our agenda has not been to reinvent a general overview of geriatric rheumatology but rather to hone in on pragmatic as well as up and coming musculoskeletal issues facing rheumatologists.
\end{abstract}

Arthritis-related disability can reflect either aging into disability or aging with disability. ${ }^{2}$ In these situations, symptom management commonly involves specialists such as rheumatologists. Among the identified unmet needs of rheumatology are (a) better provider education in clinical management, and (b) "improved understanding of targeting of specific therapies." ${ }^{3}$ Moreover, it has been over a decade since van Lankveld, Franssen, and Stenger called for "a gerontorheumatological service aimed at patients with musculoskeletal conditions." 4 To these ends, we believe the time is right to usher forward the efforts to bridge rheumatology and gerontology by crafting this issue of the Rheumatic Disease Clinics of North America.

The choice of contributors herein reflects our bias that not only rheumatologists but also other academicians who are involved in the rheumatologic aspects of aging are well suited to address the impact of musculoskeletal issues affecting function and mobility. Hence, within these pages, you will find not only clinical pearls helping the practitioner to navigate underappreciated aspects of pharmacotherapeutics but also, for example, a nuanced rheumatologic perspective on sarcopenia and an in-depth review of immune dysregulation in aging.

As guest editors, we have encouraged broad thinking among our authorship. Our contributors have been tasked not to lose sight of the principle of aging in place while at the same time aiming to drive forward thoughtful discussion within the medical community. ${ }^{5}$ Furthermore, we have encouraged our contributors to remain grounded and to keep sight of the pragmatic and clinical consequences of their respective areas of expertise. Therefore, 
interspersed among these pages may be found both philosophical perspectives and potential policy-shaping research agendas. As such, we hope this issue serves to further the study of quality of life in an aging demographic. Finally, we hope that by invoking rheumatologic insight we have broadened the clinical horizon sufficiently so as to challenge the perspectives of educators, practitioners, and academicians, alike.

\section{Biographies}

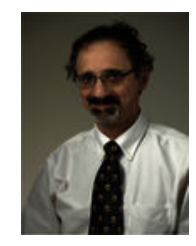

James D. Katz, MD

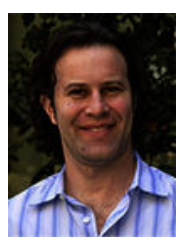

Brian Walitt, MD, MPH

\section{REFERENCES}

1. Katz JD, Walitt B . Rheumatic diseases in older adults. Clin Geriatr Med 2017;33(1):ix-x.

2. Choi S Midlife adults with functional limitations: comparison of adults with early-and late-onset arthritis-related disability. Disabil Health J 2018;11(3):374-81. [Epub ahead of print].29307574

3. Winthrop KL, Strand V, van der Heijde D, et al. The unmet need in rheumatology: reports from the targeted therapies meeting 2017. Clin Immunol 2018;186:87-93.28811201

4. van Lankveld W, Franssen M, Stenger A . Gerontorheumatology: the challenge to meet health-care demands for the elderly with musculoskeletal conditions. Rheumatology (Oxford) 2005;44(4):419_ 22.15695295

5. Morley JE . Aging successfully: the key to aging in place. J Am Med Dir Assoc 2015;16(12):10057.26545865 